\title{
Short communication Optimizing the implementation of future treatment using surrogate end-points
}

\author{
Mitch Dowsett
}

Academic Department of Biochemistry, Royal Marsden Hospital, Fulham Road, London SW3 6JJ, UK

Corresponding author: Mitch Dowsett, mitch.dowsett@icr.ac.uk

Published: 18 December 2008

This article is online at http://breast-cancer-research.com/content/10/S4/S26 (c) 2008 BioMed Central Ltd

\section{Introduction}

The word 'surrogate' is equivalent to 'substitute', and strictly a surrogate end-point should be able to substitute completely for the end-point of interest; there is, however, much misuse of the term. This short communication discusses a number of end-points that appear to have a useful role to play in the development of new agents, but even they could not be strictly called surrogates and may be better described as intermediate end-points.

The reason to seek these intermediates is that they generally provide earlier information and are easier and cheaper to use. They are also frequently more ethical to obtain, achieve or monitor than the clinical end-point, and in addition there are circumstances in which the surrogate end-point may be superior to the clinical end-point, in that it assesses the underlying disease process in a more direct manner [1]. It is argued below that change in proliferation may be one such end-point in relation to estimating clinical benefit from new agents.

Certain dangers must be considered in the application of supposed surrogates, in that these may not always reflect the disease process despite all evidence to date indicating that they do. In addition, the end-point will very often not (and maybe cannot) capture the overall risk/benefit of a treatment, but this will normally be readily apparent.

\section{Clinical trial design in early breast cancer}

The contemporary performance of adjuvant trials in early breast cancer requires inclusion of thousands of patients and years of follow up to deliver the hoped for changes in recurrence-free survival and overall survival. An example is the ATAC (Arimidex, Tamoxifen, Alone or in Combination) trial, which randomly assigned 9,366 patients with follow-up for 2.5 years before the first data were available to indicate that
Breast Cancer Research 2008, 10(Suppl 4):S26 (doi:10.1186/bcr2186)

anastrozole was more effective than either tamoxifen or the combination [2].

The neoadjuvant IMPACT (Immediate Preoperative 'Arimidex' [anastrozole], Tamoxifen, or Arimidex Combined with Tamoxifen) trial was designed to determine whether either clinical response or change in the proliferative biomarker Ki67 could predict the outcome of the ATAC trial. IMPACT required only 330 patients and follow-up of just 12 weeks to provide its primary end-points [3]. To ensure its close representation of the ATAC trial, eligibility criteria for patients were as close as possible to those employed within the adjuvant trial itself. It was observed that the change in the intermediate endpoint Ki67, at both 2 weeks and 12 weeks in IMPACT, was greater for anastrozole than for either tamoxifen or the combination, with the latter two treatment arms exhibiting very similar change in proliferation to one another. In contrast, the data on clinical response indicated no significant difference between the three arms. Thus, change in the biomarker Ki67, but not change in tumour size (clinical response), could be seen to be predictive of the outcome of the adjuvant trial. Although it is highly unlikely that adjuvant trials could ever be replaced by such neoadjuvant studies, it is possible that, by their conduct early in drug development, treatment arms that do not merit assessment in the adjuvant setting (such as the combination arm of ATAC proved to be) could be excluded and allow earlier completion of the trials.

\section{Relationship between change in Ki67 and response/benefit from treatment}

The question of whether change in Ki67 relates closely to clinical response is one that is frequently asked. In IMPACT there was a statistically significant association between the change in Ki67 and objective response when the latter is expressed in the five categories of progressive disease, no change, minimal response, partial response and complete

ATAC $=$ Arimidex, Tamoxifen, Alone or in Combination; IMPACT = Immediate Preoperative 'Arimidex' (anastrozole), Tamoxifen, or Arimidex Combined with Tamoxifen. 
response [3]. However, the relationship is very weak. Nevertheless, the argument can be made that the change in Ki67 would not be expected to act as a good surrogate for clinical response, and may in fact be rationalized to be a better indicator of benefit from treatment than clinical response itself, as follows.

In a slow growing tumour the application of an effective treatment would be expected to reduce proliferation and to lead to shrinkage of the tumour. The same effect on proliferation in a faster growing tumour may lead to slower growth and therefore treatment benefit, but this might be observed and recorded clinically as progressive disease. This would create a complete disconnect between the change in Ki67 and clinical response. However, the application of the same treatment in the adjuvant setting would be expected to lead to improved recurrence-free survival for both types of tumour. This argument suggests that certain biomarkers would be expected to be better markers of tumour efficacy than clinical response.

\section{Change in proliferation and long-term outcome}

Pretreatment Ki67, change in Ki67 and on-treatment value of Ki67 with endocrine treatment are all highly variable and indicate that biological response to such treatment is a continuum. Indeed, although we frequently dichotomize patients as responders and nonresponders on the basis of change in tumour size, it may be more appropriate to consider them good or poor responders, because tumour size change is also a continuous variable. Given that the pretreatment value of Ki67 is a strong prognostic factor and that the arguments above indicate that the change in Ki67 is indicative of benefit from therapy, the 2-week value might be expected to be an indicator of the patient's prognosis on that particular treatment. Further data from the IMPACT Trial supports this view, with the recurrence-free survival of patients being more closely predicted by 2-week Ki67 values than pretreatment values [4]. This has been one of the drivers behind the development of the POETIC (Perioperative Endocrine Treatment for Individualized Care) trial, which will test whether the greater prognostic value of on-treatment measurement of Ki67 can be confirmed in a larger patient population and be worthy of regular incorporation into a patient's disease management.

\section{Measurement of proliferation}

For a biomarker to be incorporated into clinical practice, its measurement must be sufficiently precise. We have determined that in pairs of samples taken from a single tumour at the same time using $14 \mathrm{~g}$ core-cut needles, the variability in Ki67 would require an approximately 50\% difference between two samples for these to be deemed significantly different from one another with 95\% confidence [5]. Similar analyses, but with a 2-week gap between biopsies, indicated that there was similar precision and no systematic difference between the prior and 2-week sample; this was important to establish, given the possibility that the taking of the first biopsy might itself have resulted in altered proliferation in the subsequent biopsy.

The $14 \mathrm{~g}$ core-cut biopsies taken in most of our studies may contain a million or more malignant cells, in contrast to the 1,000 cells that we routinely score in a single section; this much larger cell population might be assessed by molecular means in an extract of the core-cut, and this would theoretically give much greater analytical precision. It remains to be determined whether the mixed cell population that is sampled in an extract would give an acceptable estimate of the proliferation of tumour cells.

\section{When to measure}

The mean change in Ki67 at 2 weeks after the start of aromatase inhibitor or tamoxifen therapy is very similar overall to that after 12 weeks of treatment [6]. However, there is clear recovery in proliferation levels in a minority of the tumours, and this appears to be correlated with increased levels of HER-2 (human epidermal growth factor receptor-2) in the pretreatment sample, suggesting that this may represent early acquired resistance to the endocrine agent. If this can be proven, it provides an important extension of the use of neoadjuvant endocrine therapy; in the past we have rejected the study of acquired resistance in the neoadjuvant model because clinical progression rarely occurs or is allowed to occur before surgery. The application of this surrogate biomarker therefore potentially opens the neoadjuvant arena, first to the biological study of acquired resistance and second to the application of agents with the aim of avoiding this. This type of rationale underpinned the design of the anastrozole with/without gefitinib study [7], in which one arm received gefitinib after an initial 2 weeks of anastrozole alone. Unfortunately, the apparently poor clinical responsiveness to gifitinib in this population meant that the hypothetical use of the model in this manner was not effectively tested.

\section{Does on-treatment Ki67 also predict long-term outcome after chemotherapy?}

We recently extended our analyses of on-treatment measurement of Ki67 to the estimation of long-term outcome with chemotherapy [8]. In these studies only those patients who failed to achieve a pathological complete remission could be evaluated because estimates of Ki67 in the excision biopsy were required. Although patients with high Ki67 pretreatment responded better to the neoadjuvant chemotherapy, these high pretreatment values indicated a worse outcome for high Ki67. Of particular note, measurement of Ki67 in the excision sample exhibited much better prediction of outcome, with $50 \%$ of patients in the highest tertile of Ki67 having relapsed within 18 months, whereas more than $50 \%$ of patients in the lower two tertiles had more than 7 years of relapse-free survival. To make this observation clinically valuable within the neoadjuvant chemotherapy setting, such measurement of 
Ki67 would necessarily require earlier measurement than at excision. As yet, no studies have linked earlier measurement of Ki67 after starting chemotherapy with long-term outcome.

\section{Conclusion}

The measurement of on-treatment proliferation using Ki67 provides a useful tool for speeding development of new drugs. However, although the principle of on-treatment measurement is likely to remain solid, the end-point for judging effectiveness is likely to vary markedly between agents.

\section{Competing interests}

The author has received honoraria for advice and lectures as well as grant income from AstraZeneca and Novartis during the past 5 years.

\section{Acknowledgements}

I am grateful to the very large number of investigators, both clinical and scientific, who have contributed to these studies over many years.

This article has been published as part of Breast Cancer Research Volume 10 Supplement 4, 2008: Controversies in Breast Cancer 2008. The full contents of the supplement are available online at http://breast-cancer-research.com/supplements/10/S4

\section{References}

1. Lassere MN: The Biomarker-Surrogacy Evaluation Schema: a review of the biomarker-surrogate literature and a proposal for a criterion-based, quantitative, multidimensional hierarchical levels of evidence schema for evaluating the status of biomarkers as surrogate endpoints. Stat Methods Med Res 2008, 17:303-340.

2. Baum M, Budzar AU, Cuzick J, Forbes J, Houghton JH, Klijn JG, Sahmoud T; ATAC Trialists' Group: Anastrozole alone or in combination with tamoxifen versus tamoxifen alone for adjuvant treatment of postmenopausal women with early breast cancer: first results of the ATAC randomised trial. Lancet 2002, 359:2131-2139.

3. Dowsett M, Smith IE, Ebbs SR, Dixon JM, Skene A, Griffith C, Boeddinghaus I, Salter J, Detre S, Hills M, Ashley S, Francis S, Walsh G; IMPACT Trialists: Short-term changes in Ki-67 during neoadjuvant treatment of primary breast cancer with anastrozole or tamoxifen alone or combined correlate with recurrence-free survival. Clin Cancer Res 2005, 11:951s-958s.

4. Dowsett M, Smith IE, Ebbs SR, Dixon JM, Skene A, A'Hern R, Salter J, Detre S, Hills M, Walsh G; IMPACT Trialists Group: Prognostic value of Ki67 expression after short-term presurgical endocrine therapy for primary breast cancer. J Natl Cancer Inst 2007, 99:167-170.

5. Ellis PA, Smith IE, Detre S, Burton SA, Salter J, A'Hern R, Walsh G, Johnston SR, Dowsett M: Reduced apoptosis and proliferation and increased $\mathrm{Bcl}-2$ in residual breast cancer following preoperative chemotherapy. Breast Cancer Res Treat 1998, 48:107-116.

6. Dowsett M, Ebbs SR, Dixon JM, Skene A, Griffith C, Boeddinghaus I, Salter J, Detre S, Hills M, Ashley S, Francis S, Walsh G, Smith IE: Biomarker changes during neoadjuvant anastrozole, tamoxifen, or the combination: influence of hormonal status and HER-2 in breast cancer-a study from the IMPACT trialists. J Clin Oncol 2005, 23:2477-2492.

7. Smith IE, Walsh G, Skene A, Llombart A, Mayordomo Jl, Detre S, Salter J, Clark E, Magill P, Dowsett M: A phase II placebo-controlled trial of neoadjuvant anastrozole alone or with gefitinib in early breast cancer. J Clin Oncol 2007, 25:3816-3822.

8. Jones RL, Salter J, A'hern R, Nerurkar A, Parton M, Reis-Filho JS, Smith IE, Dowsett M: The prognostic significance of Ki67 before and after neoadjuvant chemotherapy in breast cancer. Breast Cancer Res Treat 2008 [Epub ahead of print]. 